\title{
Solvability of a $q$-fractional integral equation arising in the study of an epidemic model
}

\section{Mohamed Jleli and Bessem Samet ${ }^{*}$}

\section{"Correspondence:}

bsamet@ksu.edu.sa

Department of Mathematics,

College of Science, King Saud

University, P.O. Box 2455, Riyadh,

11451, Saudi Arabia

\begin{abstract}
We study the solvability of a functional equation involving $q$-fractional integrals. Such an equation arises in the study of the spread of an infectious disease that does not induce permanent immunity. Our method is based on the noncompactness measure argument in a Banach algebra and an extension of Darbo's fixed point theorem.
\end{abstract}

MSC: 62P10; 26A33; 45G10

Keywords: epidemic model; 9 -calculus; fractional order; measure of noncompactness

\section{Introduction}

In this paper, we deal with the solvability of the $q$-fractional integral equation

$$
x(t)=\prod_{i=1}^{N}\left(f_{i}(t)+\frac{g_{i}(t, x(t))}{\Gamma_{q}\left(\alpha_{i}\right)} \int_{0}^{t}(t-q s)^{\left(\alpha_{i}-1\right)} u_{i}(s, x(s)) d_{q} s\right), \quad t \in I,
$$

where $I=[0,1], q \in(0,1), \alpha_{i}>1, f_{i}: I \rightarrow \mathbb{R}$, and $g_{i}, u_{i}: I \times \mathbb{R} \rightarrow \mathbb{R}(i=1, \ldots, N)$.

For $N=1, q=1$, and $\alpha_{1}=1$, equation (1.1) arises in the study of the spread of an infectious disease that does not induce permanent immunity (see [1-3]).

Equation (1.1) can be written as

$$
x(t)=\prod_{i=1}^{N}\left(f_{i}(t)+g_{i}(t, x(t)) I_{q}^{\alpha_{i}} u_{i}(\cdot, x(\cdot))(t)\right), \quad t \in I,
$$

where $I_{q}^{\alpha_{i}}$ is the $q$-fractional integral of order $\alpha_{i}$ defined by [4]

$$
I_{q}^{\alpha_{i}} h(t)=\frac{1}{\Gamma_{q}\left(\alpha_{i}\right)} \int_{0}^{t}(t-q s)^{\left(\alpha_{i}-1\right)} h(s) d_{q} s, \quad t \in I .
$$

Via noncompactness measure argument in a Banach algebra, we provide sufficient conditions for the existence of at least one solution to equation (1.1). We also give an example in order to illustrate our existence result.

(c) The Author(s) 2017. This article is distributed under the terms of the Creative Commons Attribution 4.0 International License (http://creativecommons.org/licenses/by/4.0/), which permits unrestricted use, distribution, and reproduction in any medium, provided you give appropriate credit to the original author(s) and the source, provide a link to the Creative Commons license, and indicate if changes were made. 
The concept of noncompactness measure was used by many authors in order to study the solvability of various classes of integral equations (see [5-15] and references therein). Very recently, Jleli, Mursaleen, and Samet [16] studied the solvability of a functional equation involving the $q$-fractional integral equation

$$
x(t)=F\left(t, x(a(t)), \frac{f(t, x(t))}{\Gamma_{q}(\alpha)} \int_{0}^{t}(t-q s)^{(\alpha-1)} u(s, x(s)) d_{q} s\right), \quad t \in[0,1] .
$$

Using the noncompactness measure tool, the authors obtained an existence result for such an equation. To the best of our knowledge, [16] is the only work dealing with the solvability of a $q$-fractional integral equation with noncompactness measure. In this paper, we use a different approach in order to establish our existence result. We first establish a fixed point theorem in a Banach algebra via a measure of noncompactness satisfying condition $(m)$. Next, via the obtained fixed point result, we prove that, under certain conditions, equation (1.1) has at least one solution.

\section{Preliminaries on quantum calculus}

The concept of quantum calculus was introduced by Jackson $[17,18]$. This subject is rich in history and has many applications (see [19-26]). In this section, we recall some basic facts on quantum calculus and present additional properties that will be used later. For more details, we refer to [21].

Let $q \in[0, \infty) \backslash\{1\}$. For $x \in \mathbb{R}$, we define the $q$-real number $[x]_{q}$ by

$$
[x]_{q}=\frac{1-q^{x}}{1-q} .
$$

The $q$-factorial of $x$ is defined by

$$
(x, q)_{0}=1, \quad(x, q)_{k}=\prod_{i=0}^{k-1}\left(1-x q^{i}\right), \quad k=1,2, \ldots, \infty .
$$

For $(a, b) \in \mathbb{R}^{2}$, the $q$-analog of $(a-b)^{k}$ is defined by

$$
(a-b)^{(0)}=1, \quad(a-b)^{(k)}=\prod_{i=0}^{k-1}\left(a-q^{i} b\right), \quad k=1,2, \ldots
$$

For $\beta \in \mathbb{R},(a, b) \in \mathbb{R}^{2}$, and $a \geq 0$,

$$
(a-b)^{(\beta)}=a^{\beta} \prod_{i=0}^{\infty} \frac{a-b q^{i}}{a-b q^{\beta+i}} .
$$

Note that, for $b=0$, we have

$$
a^{(\beta)}=a^{\beta} .
$$

Lemma 2.1 ([27]) If $\beta>0$ and $0 \leq a \leq b \leq t$, then

$$
(t-b)^{(\beta)} \leq(t-a)^{(\beta)} .
$$


The $q$-gamma function is given by

$$
\Gamma_{q}(x)=\frac{(1-q)^{(x-1)}}{(1-q)^{x-1}}, \quad x \notin\{0,-1,-2, \ldots\} .
$$

We have the following property:

$$
\Gamma_{q}(x+1)=[x]_{q} \Gamma_{q}(x)
$$

Let $f:[0, T] \rightarrow \mathbb{R}$ be a given function $(T>0)$. We define the $q$-integral of the function $f$ by

$$
I_{q} f(t)=\int_{0}^{t} f(s) d_{q} s=t(1-q) \sum_{n=0}^{\infty} f\left(t q^{n}\right) q^{n}, \quad t \in[0, T] .
$$

If $\eta \in[0, T]$, then

$$
\int_{\eta}^{T} f(s) d_{q} s=\int_{0}^{T} f(s) d_{q} s-\int_{0}^{\eta} f(s) d_{q} s
$$

Lemma 2.2 Let $f:[0,1] \rightarrow \mathbb{R}$ be a continuous function. Then

$$
\left|\int_{0}^{t} f(s) d_{q} s\right| \leq \int_{0}^{t}|f(s)| d_{q} s, \quad t \in[0,1]
$$

Remark 2.3 If $0 \leq t_{1} \leq t_{2} \leq 1$ and $f:[0,1] \rightarrow \mathbb{R}$ is a continuous function, then the inequality

$$
\left|\int_{t_{1}}^{t_{2}} f(s) d_{q} s\right| \leq \int_{t_{1}}^{t_{2}}|f(s)| d_{q} s
$$

is not satisfied in general. As a counterexample, we refer to [21], p.12.

Let $f:[0,1] \rightarrow \mathbb{R}$ be a given function. The fractional $q$-integral of order $\alpha \geq 0$ of the function $f$ is given by $I_{q}^{0} f(t)=f(t)$ and

$$
I_{q}^{\alpha} f(t)=\frac{1}{\Gamma_{q}(\alpha)} \int_{0}^{t}(t-q s)^{(\alpha-1)} f(s) d q_{s}, \quad t \in[0,1], \alpha>0 .
$$

Note that, for $\alpha=1$, we have

$$
I_{q}^{1} f(t)=I_{q} f(t), \quad t \in[0,1]
$$

For $f \equiv 1$,

$$
I_{q}^{\alpha} 1(t)=\frac{1}{\Gamma_{q}(\alpha+1)} t^{\alpha}, \quad t \in[0,1]
$$




\section{A fixed point theorem in a Banach algebra via a measure of noncompactness satisfying condition $(m)$}

In this section, we recall the axiomatic approach of noncompactness measure introduced by Banas and Goebel [10]. Next, we establish a fixed point theorem in a Banach algebra via a measure of noncompactness satisfying condition $(m)$. This fixed point result plays an important rule in the proof of our existence result.

Let $(E, \cdot)$ be a Banach Algebra over $\mathbb{R}$ with respect to a certain norm $\|\cdot\|_{E}$. We denote by $0_{E}$ the zero vector of $E$. For $x \in E$ and $r>0$, we denote by $B(x, r)$ the open ball in $E$ of center $x$ and radius $r$, that is,

$$
B(x, r)=\left\{y \in E:\|x-y\|_{E}<r\right\}
$$

We denote by $\mathcal{P}(E)$ the set of all nonempty subsets of $E$. If $M \in \mathcal{P}(E)$, then the symbol $\bar{M}$ denotes the closure of $M$. The symbol $\operatorname{Conv}(M)$ stands for the convex hull of $M$. For $(M, N) \in \mathcal{P}(E) \times \mathcal{P}(E)$ and $\alpha \in \mathbb{R}$, we denote

$$
M+N=\{x+y:(x, y) \in M \times N\}
$$

and

$$
\alpha M=\{\alpha x: x \in M\}
$$

We denote by $\mathcal{P}_{b}(E)$ the set of all nonempty bounded subsets of $E$. For $M \in \mathcal{P}_{b}(E)$, we denote by $\|M\|$ the norm of $M$, that is,

$$
\|M\|=\sup \left\{\|x\|_{E}: x \in M\right\} .
$$

We denote by $\mathcal{P}_{r c}(E)$ the set of all relatively compact subsets of $E$. For $M, N \in \mathcal{P}(E)$, we denote by $M N$ the product set

$$
M N=\{x \cdot y:(x, y) \in M \times N\} .
$$

In what follows, we recall the axiomatic approach of a measure of noncompactness introduced by Banas and Goebel [10].

Definition 3.1 Let $\mu: \mathcal{P}_{b}(E) \rightarrow[0, \infty)$ be a given mapping. We say that $\mu$ is a measure of noncompactness in $E$ if it satisfies the following axioms:

(A1) The family $\operatorname{ker} \mu=\mu^{-1}(\{0\})$ is a subset of $\mathcal{P}_{r c}(E)$.

(A2) $(M, N) \in \mathcal{P}_{b}(E) \times \mathcal{P}_{b}(E), M \subset N \Longrightarrow \mu(M) \leq \mu(N)$.

(A3) $\mu(\bar{M})=\mu(M), M \in \mathcal{P}_{b}(E)$.

(A4) $\mu(\operatorname{Conv}(M))=\mu(M), M \in \mathcal{P}_{b}(E)$.

(A5) $\mu(\lambda M+(1-\lambda) N) \leq \lambda \mu(M)+(1-\lambda) \mu(N)$ for $\lambda \in[0,1]$ and $(M, N) \in \mathcal{P}_{b}(E) \times \mathcal{P}_{b}(E)$.

(A6) If $\left\{M_{n}\right\}$ is a sequence of closed sets from $\mathcal{P}_{b}(E)$ such that $M_{n+1} \subset M_{n}$ for $n=1,2, \ldots$ and if $\lim _{n \rightarrow \infty} \mu\left(M_{n}\right)=0$, then the intersection set $M_{\infty}=\bigcap_{n=1}^{\infty} M_{n}$ is nonempty.

Now, let us recall the following important result, which is called Drabo's fixed point theorem $[10,28]$. 
Lemma 3.2 Let D be a nonempty, bounded, closed, and convex subset of $E$, and let $T: D \rightarrow$ $D$ be a continuous mapping. Suppose that there exists a constant $k \in(0,1)$ such that

$$
\mu(T M) \leq k \mu(M)
$$

for any nonempty subset $M$ of $D$, where $\mu$ is a measure of noncompactness in $E$. Then $T$ has at least one fixed point in $D$.

The following concept was introduced by Banas and Olszowy [13].

Definition 3.3 Let $\mu$ be a measure of noncompactness in $E$. We say that $\mu$ satisfies condition $(m)$ if

$$
\mu(M N) \leq\|M\| \mu(N)+\|N\| \mu(M), \quad(M, N) \in \mathcal{P}_{b}(E) \times \mathcal{P}_{b}(E) .
$$

Lemma 3.4 Let $\mu$ be a measure of noncompactness in E satisfying condition (m). Let $\left\{M_{i}\right\}_{i=1, \ldots, q}$ be a finite sequence in $\mathcal{P}_{b}(E), q \geq 2$. Then

$$
\mu\left(\prod_{i=1}^{q} M_{i}\right) \leq \sum_{i=1}^{q} \prod_{j=1, j \neq i}^{q}\left\|M_{j}\right\| \mu\left(M_{i}\right) .
$$

Proof We shall use the induction principle. For $q=2$, (3.1) follows immediately from Definition 3.3. Suppose now that (3.1) is satisfied for some $q \geq 2$. We have to prove that

$$
\mu\left(\prod_{i=1}^{q+1} M_{i}\right) \leq \sum_{i=1}^{q+1} \prod_{j=1, j \neq i}^{q+1}\left\|M_{j}\right\| \mu\left(M_{i}\right) .
$$

Using (3.1) and Definition 3.3, we have

$$
\begin{aligned}
\mu\left(\prod_{i=1}^{q+1} M_{i}\right) & =\mu\left(\left(\prod_{i=1}^{q} M_{i}\right) M_{q+1}\right) \\
& \leq \mu\left(\prod_{i=1}^{q} M_{i}\right)\left\|M_{q+1}\right\|+\mu\left(M_{q+1}\right)\left\|\prod_{i=1}^{q} M_{i}\right\| \\
& \leq \mu\left(\prod_{i=1}^{q} M_{i}\right)\left\|M_{q+1}\right\|+\mu\left(M_{q+1}\right) \prod_{j=1}^{q}\left\|M_{j}\right\| \\
& \leq \sum_{i=1}^{q} \prod_{j=1, j \neq i}^{q}\left\|M_{j}\right\| \mu\left(M_{i}\right)\left\|M_{q+1}\right\|+\mu\left(M_{q+1}\right) \prod_{j=1}^{q}\left\|M_{j}\right\| \\
& =\sum_{i=1}^{q} \prod_{j=1, j \neq i}^{q+1}\left\|M_{j}\right\| \mu\left(M_{i}\right)\left\|M_{q+1}\right\|+\mu\left(M_{q+1}\right) \prod_{j=1}^{q}\left\|M_{j}\right\| \\
& =\sum_{i=1}^{q+1} \prod_{j=1, j \neq i}^{q+1}\left\|M_{j}\right\| \mu\left(M_{i}\right) .
\end{aligned}
$$

Thus, we proved (3.2). Finally, (3.1) follows by the induction principle. 
Now, we deal with the fixed point problem

$$
\left\{\begin{array}{l}
\text { Find } x \in D \text { such that } \\
x=\prod_{i=1}^{N} T_{i} x
\end{array}\right.
$$

where $D \in \mathcal{P}(E)$ and $T_{i}: D \rightarrow E, i=1, \ldots, N, N \geq 1$, are given operators.

We have the following fixed point result.

Theorem 3.5 Assume that D is nonempty, bounded, closed, and convex subset of the Banach algebra E. Assume also that the following conditions are satisfied:

(i) $T_{i}$ is continuous, $i=1, \ldots, N$.

(ii) $T_{i} D$ is bounded, $i=1, \ldots, N$.

(iii) $T D \subset D$, where $T x=\prod_{i=1}^{N} T_{i} x$.

(iv) There exists a finite sequence $\left\{k_{i}\right\}_{i=1}^{q} \subset(0, \infty)$ such that

$$
\mu\left(T_{i} M\right) \leq k_{i} \mu(M), \quad i=1, \ldots, N
$$

for any nonempty subset $M$ of $D$, where $\mu$ is a measure of noncompactness in $E$ satisfying condition $(m)$.

(v) $\sum_{i=1}^{q} k_{i} \prod_{j=1, j \neq i}^{q}\left\|T_{j} D\right\|<1$.

Then problem (3.3) has at least one solution in $D$.

Proof Let $M$ be nonempty subset of $D$. Using Lemma 3.4 and the considered assumptions, we obtain

$$
\begin{aligned}
\mu(T M) & =\mu\left(\prod_{i=1}^{N} T_{i} M\right) \leq \sum_{i=1}^{N} \prod_{j=1, j \neq i}^{N}\left\|T_{j} M\right\| \mu\left(T_{i} M\right) \\
& \leq \sum_{i=1}^{N} k_{i} \prod_{j=1, j \neq i}^{N}\left\|T_{j} M\right\| \mu(M) \leq\left(\sum_{i=1}^{N} k_{i} \prod_{j=1, j \neq i}^{N}\left\|T_{j} D\right\|\right) \mu(M) .
\end{aligned}
$$

Thus, we have proved that

$$
\mu(T M) \leq k \mu(M)
$$

for every nonempty subset $M$ of $D$, where

$$
k=\sum_{i=1}^{N} k_{i} \prod_{j=1, j \neq i}^{N}\left\|T_{j} D\right\|<1 .
$$

The result follows by Lemma 3.2.

Remark 3.6 For $N=2$, Theorem 3.5 reduces to a fixed point theorem established in [13].

Remark 3.7 For $N=1$, Theorem 3.5 reduces to Lemma 3.2. 


\section{Main result}

In this section, we state and prove our main result concerning the existence of solutions to equation (1.1).

Let $E=C(I ; \mathbb{R})$ be the Banach space of all real-valued continuous functions in $I$ equipped with the norm

$$
\|u\|_{E}=\max \{|u(t)|: t \in I\}, \quad u \in E
$$

Clearly, $E$ is a Banach algebra with respect to the operation - defined by

$$
(u \cdot v)(t)=u(t) v(t), \quad t \in I,(u, v) \in E \times E .
$$

Let $M \in \mathcal{P}_{b}(E)$. For $x \in M$ and $\varepsilon>0$, set

$$
\begin{aligned}
& \omega(x, \varepsilon)=\sup \{|x(t)-x(s)|: t, s \in I,|t-s| \leq \varepsilon\}, \\
& \omega(M, \varepsilon)=\sup \{\omega(x, \varepsilon): x \in M\} .
\end{aligned}
$$

It was proved in $[10]$ that the mapping $\mu: \mathcal{P}_{b}(E) \rightarrow[0, \infty)$ defined by

$$
\mu(M)=\lim _{\varepsilon \rightarrow 0^{+}} \omega(M, \varepsilon), \quad M \in \mathcal{P}_{b}(E)
$$

is a measure of noncompactness in $E$. Moreover, $\mu$ satisfies condition (m) (see [13]).

Equation (1.1) can be written as

$$
x=T x=\prod_{i=1}^{N} T_{i} x,
$$

where fo,r $i=1, \ldots, N$,

$$
\left(T_{i} x\right)(t)=f_{i}(t)+\frac{g_{i}(t, x(t))}{\Gamma_{q}\left(\alpha_{i}\right)} \int_{0}^{t}(t-q s)^{\left(\alpha_{i}-1\right)} u_{i}(s, x(s)) d_{q} s, \quad t \in I .
$$

We consider the following assumption:

(A1) For $i=1, \ldots, N$, the functions $f_{i}: I \rightarrow \mathbb{R}$ and $g_{i}, u_{i}: I \times \mathbb{R} \rightarrow \mathbb{R}$ are continuous.

Lemma 4.1 For every $i=1, \ldots, N$, the operator $T_{i}: E \rightarrow E$ is well defined.

Proof Fix $i \in\{1, \ldots, N\}$. We have just to prove that the operator

$$
\left(S_{i} x\right)(t)=\int_{0}^{t}(t-q s)^{\left(\alpha_{i}-1\right)} u_{i}(s, x(s)) d_{q} s, \quad t \in I,
$$

maps $E$ into it self. To do this, let us fix $x \in E$. For all $t \in I$, we have

$$
\begin{aligned}
\left(S_{i} x\right)(t) & =t(1-q) \sum_{n=0}^{\infty} q^{n}\left(t-q^{n+1} t\right)^{\left(\alpha_{i}-1\right)} u_{i}\left(t q^{n}, x\left(t q^{n}\right)\right) \\
& =t^{\alpha_{i}}(1-q) \sum_{n=0}^{\infty} q^{n}\left(1-q^{n+1}\right)^{\left(\alpha_{i}-1\right)} u_{i}\left(t q^{n}, x\left(t q^{n}\right)\right) .
\end{aligned}
$$


Since $q^{n+1} \in(0,1)$, by Lemma 2.1 we have

$$
\left(1-q^{n+1}\right)^{\left(\alpha_{i}-1\right)} \leq(1-0)^{\left(\alpha_{i}-1\right)}=1
$$

Therefore, the continuity of $u_{i}$ and the Weierstrass convergence theorem give us the desired result.

We suppose also that the following assumptions are satisfied:

(A2) For all $i=1, \ldots, N$, there exist constants $C_{i}>0$ and $d_{i}>0$ such that

$$
\left|g_{i}(t, x)-g_{i}(t, y)\right| \leq C_{i}|x-y|^{d_{i}}, \quad(t, x, y) \in I \times I \times \mathbb{R}
$$

(A3) For all $i=1, \ldots, N$, there exist a constant $e_{i}>0$ and a nondecreasing continuous function $\varphi_{i}:[0, \infty) \rightarrow[0, \infty)$ such that

$$
\left|u_{i}(t, x)-u_{i}(t, y)\right| \leq \varphi_{i}\left(|x-y|^{e_{i}}\right), \quad(t, x, y) \in I \times I \times \mathbb{R} .
$$

(A4) For all $i=1, \ldots, N$, we have

$$
u_{i}(t, 0)=0, \quad t \in I \text {. }
$$

(A5) There exists $r>0$ such that

$$
\left\|f_{i}\right\|_{E}+\frac{\left(C_{i} r^{d_{i}}+g_{i}^{*}\right) \varphi_{i}\left(r^{e_{i}}\right)}{\Gamma_{q}\left(\alpha_{i}+1\right)} \leq r^{1 / N}
$$

where

$$
g_{i}^{*}=\max \left\{\left|g_{i}(t, 0)\right|: t \in I\right\} .
$$

Lemma 4.2 Under Assumptions (A1)-(A5), for all $i=1, \ldots, N$, we have

$$
T_{i} \overline{B\left(0_{E}, r\right)} \subset \overline{B\left(0_{E}, r^{1 / N}\right)}
$$

Proof Let $i \in\{1, \ldots, N\}$ be fixed, and let $x \in \overline{B\left(0_{E}, r\right)}$. For all $t \in I$, we have

$$
\begin{aligned}
\left|\left(T_{i} x\right)(t)\right| & \leq\left|f_{i}(t)\right|+\frac{\left|g_{i}(t, x(t))\right|}{\Gamma_{q}\left(\alpha_{i}\right)} \int_{0}^{t}(t-q s)^{\left(\alpha_{i}-1\right)}\left|u_{i}(s, x(s))\right| d_{q} s \\
& \leq\left\|f_{i}\right\|_{E}+\frac{\left|g_{i}(t, x(t))\right|}{\Gamma_{q}\left(\alpha_{i}\right)} \int_{0}^{t}(t-q s)^{\left(\alpha_{i}-1\right)}\left|u_{i}(s, x(s))\right| d_{q} s \\
& \leq\left\|f_{i}\right\|_{E}+\frac{\left|g_{i}(t, x(t))-g_{i}(t, 0)\right|+\left|g_{i}(t, 0)\right|}{\Gamma_{q}\left(\alpha_{i}\right)} \int_{0}^{t}(t-q s)^{\left(\alpha_{i}-1\right)}\left|u_{i}(s, x(s))\right| d_{q} s .
\end{aligned}
$$

Using Assumption (A2), we obtain

$$
\left|g_{i}(t, x(t))-g_{i}(t, 0)\right|+\left|g_{i}(t, 0)\right| \leq C_{i} r^{d_{i}}+g_{i}^{*},
$$


By Assumptions (A3) and (A4) we have

$$
\left|u_{i}(s, x(s))\right|=\left|u_{i}(s, x(s))-u_{i}(s, 0)\right| \leq \varphi_{i}\left(r^{e_{i}}\right) .
$$

Therefore, we obtain

$$
\begin{aligned}
\left|\left(T_{i} x\right)(t)\right| & \leq\left\|f_{i}\right\|_{E}+\frac{\left(C_{i} r^{d_{i}}+g_{i}^{*}\right) \varphi_{i}\left(r^{e_{i}}\right)}{\Gamma_{q}\left(\alpha_{i}\right)} \int_{0}^{t}(t-q s)^{\left(\alpha_{i}-1\right)} d_{q} s \\
& =\left\|f_{i}\right\|_{E}+\frac{\left(C_{i} r^{d_{i}}+g_{i}^{*}\right) \varphi_{i}\left(r^{e_{i}}\right)}{\Gamma_{q}\left(\alpha_{i}+1\right)} t^{\alpha_{i}} \\
& \leq\left\|f_{i}\right\|_{E}+\frac{\left(C_{i} r^{d_{i}}+g_{i}^{*}\right) \varphi_{i}\left(r^{e_{i}}\right)}{\Gamma_{q}\left(\alpha_{i}+1\right)} .
\end{aligned}
$$

By Assumption (A5) we get

$$
\left\|T_{i} x\right\|_{E} \leq r^{1 / N}
$$

which proves the desired result.

Lemma 4.3 Under Assumptions (A1)-(A5), for all $i=1, \ldots, N$,

$$
T_{i}: \overline{B\left(0_{E}, r\right)} \rightarrow \overline{B\left(0_{E}, r^{1 / N}\right)}
$$

is continuous.

Proof Under the considered assumptions, we have just to prove that, for all $i=1, \ldots, N$, the operator $S_{i}: \overline{B\left(0_{E}, r\right)} \rightarrow E$ defined by (4.3) is continuous. To do this, let us fix $i \in\{1, \ldots, N\}$ and $\varepsilon>0$ such that $\|x-y\|_{E} \leq \varepsilon,(x, y) \in \overline{B\left(0_{E}, r\right)} \times \overline{B\left(0_{E}, r\right)}$. For all $t \in I$, we have

$$
\begin{aligned}
\left|\left(S_{i} x\right)(t)-\left(S_{i} y\right)(t)\right| & =\left|\int_{0}^{t}(t-q s)^{\left(\alpha_{i}-1\right)}\left(u_{i}(s, x(s))-u_{i}(s, y(s))\right) d_{q} s\right| \\
& \leq \int_{0}^{t}(t-q s)^{\left(\alpha_{i}-1\right)}\left|u_{i}(s, x(s))-u_{i}(s, y(s))\right| d_{q} s \\
& \leq \frac{u_{r}^{i}(\varepsilon) t^{\alpha_{i}}}{\left[\alpha_{i}\right]_{q}} \\
& \leq \frac{u_{r}^{i}(\varepsilon)}{\left[\alpha_{i}\right]_{q}}
\end{aligned}
$$

where

$$
u_{r}^{i}(\varepsilon)=\sup \left\{\left|u_{i}(t, x)-u_{i}(t, y)\right|: t \in I, x, y \in[-r, r],|x-y| \leq \varepsilon\right\} .
$$

Therefore,

$$
\left\|S_{i} x-S_{i} y\right\|_{E} \leq \frac{u_{r}^{i}(\varepsilon)}{\left[\alpha_{i}\right]_{q}}
$$


Passing to the limit as $\varepsilon \rightarrow 0$ and using the uniform continuity of $u_{i}$ on the compact set $I \times[-r, r]$, we obtain

$$
\lim _{\varepsilon \rightarrow 0} \frac{u_{r}^{i}(\varepsilon)}{\left[\alpha_{i}\right]_{q}}=0,
$$

which yields the desired result.

Next, we need the following additional assumption:

(A6) For all $i=1, \ldots, N$, there exists a constant $\lambda_{i}>0$ such that

$$
t^{d_{i}}+\varphi_{i}\left(t^{e_{i}}\right) \leq \lambda_{i} t, \quad t \geq 0
$$

Lemma 4.4 Under assumptions (A1)-(A6), for all $i=1, \ldots, N$, we have

$$
\mu\left(T_{i} M\right) \leq k_{i} \mu(M)
$$

for every nonempty subset $M$ of $\overline{B\left(0_{E}, r\right)}$, where

$$
k_{i}=\lambda_{i} \max \left\{\frac{C_{i} \varphi_{i}\left(r^{e_{i}}\right)}{\Gamma_{q}\left(\alpha_{i}+1\right)}, \frac{\left(C_{i} r^{d_{i}}+g_{i}^{*}\right)}{\Gamma_{q}\left(\alpha_{i}\right)}\right\} .
$$

Proof Fix $i \in\{1, \ldots, N\}$. Let $M$ be a nonempty subset of $\overline{B\left(0_{E}, r\right)}, \varepsilon>0$, and $x \in M$. Let $\left(t_{1}, t_{2}\right) \in I \times I$ be such that $\left|t_{1}-t_{2}\right| \leq \varepsilon$. Without loss of the generality, we may assume that $t_{1} \geq t_{2}$. Therefore, we have

$$
\begin{aligned}
\left|\left(T_{i} x\right)\left(t_{1}\right)-\left(T_{i} x\right)\left(t_{2}\right)\right| & \\
\leq & \left|f_{i}\left(t_{1}\right)-f_{i}\left(t_{2}\right)\right|+\mid \frac{g_{i}\left(t_{1}, x\left(t_{1}\right)\right)}{\Gamma_{q}\left(\alpha_{i}\right)} \int_{0}^{t_{1}}\left(t_{1}-q s\right)^{\left(\alpha_{i}-1\right)} u_{i}(s, x(s)) d_{q} s \\
& -\frac{g_{i}\left(t_{2}, x\left(t_{2}\right)\right)}{\Gamma_{q}\left(\alpha_{i}\right)} \int_{0}^{t_{2}}\left(t_{2}-q s\right)^{\left(\alpha_{i}-1\right)} u_{i}(s, x(s)) d_{q} s \mid \\
\leq & \omega\left(f_{i}, \varepsilon\right)+\mid \frac{g_{i}\left(t_{1}, x\left(t_{1}\right)\right)}{\Gamma_{q}\left(\alpha_{i}\right)} \int_{0}^{t_{1}}\left(t_{1}-q s\right)^{\left(\alpha_{i}-1\right)} u_{i}(s, x(s)) d_{q} s \\
& -\frac{g_{i}\left(t_{2}, x\left(t_{2}\right)\right)}{\Gamma_{q}\left(\alpha_{i}\right)} \int_{0}^{t_{1}}\left(t_{1}-q s\right)^{\left(\alpha_{i}-1\right)} u_{i}(s, x(s)) d_{q} s \mid \\
& +\mid \frac{g_{i}\left(t_{2}, x\left(t_{2}\right)\right)}{\Gamma_{q}\left(\alpha_{i}\right)} \int_{0}^{t_{1}}\left(t_{1}-q s\right)^{\left(\alpha_{i}-1\right)} u_{i}(s, x(s)) d_{q} s \\
& -\frac{g_{i}\left(t_{2}, x\left(t_{2}\right)\right)}{\Gamma_{q}\left(\alpha_{i}\right)} \int_{0}^{t_{2}}\left(t_{2}-q s\right)^{\left(\alpha_{i}-1\right)} u_{i}(s, x(s)) d_{q} s \mid \\
\leq & \omega\left(f_{i}, \varepsilon\right)+\frac{\left|g_{i}\left(t_{1}, x\left(t_{1}\right)\right)-g_{i}\left(t_{2}, x\left(t_{2}\right)\right)\right|}{\Gamma_{q}\left(\alpha_{i}\right)} \int_{0}^{t_{1}}\left(t_{1}-q s\right)^{\left(\alpha_{i}-1\right)}\left|u_{i}(s, x(s))\right| d_{q} s \\
& +\frac{\left|g_{i}\left(t_{2}, x\left(t_{2}\right)\right)\right|}{\Gamma_{q}\left(\alpha_{i}\right)}\left|\int_{0}^{t_{1}}\left(t_{1}-q s\right)^{\left(\alpha_{i}-1\right)} u_{i}(s, x(s)) d_{q} s-\int_{0}^{t_{2}}\left(t_{2}-q s\right)^{\left(\alpha_{i}-1\right)} u_{i}(s, x(s)) d_{q} s\right| \\
:= & \omega\left(f_{i}, \varepsilon\right)+(I)+(I I),
\end{aligned}
$$


where

$$
\omega\left(f_{i}, \varepsilon\right)=\sup \left\{\left|f_{i}(t)-f_{i}(s)\right|: t, s \in I,|t-s| \leq \varepsilon\right\} .
$$

Now, let us estimate $(I)$ and $(I I)$.

- Estimate of $(I)$. We have

$$
\frac{1}{\Gamma_{q}\left(\alpha_{i}\right)} \int_{0}^{t_{1}}\left(t_{1}-q s\right)^{\left(\alpha_{i}-1\right)}\left|u_{i}(s, x(s))\right| d_{q} s \leq \frac{\varphi_{i}\left(r^{e_{i}}\right)}{\Gamma_{q}\left(\alpha_{i}+1\right)} .
$$

On the other hand,

$$
\begin{aligned}
\left|g_{i}\left(t_{1}, x\left(t_{1}\right)\right)-g_{i}\left(t_{2}, x\left(t_{2}\right)\right)\right| & \leq\left|g_{i}\left(t_{1}, x\left(t_{1}\right)\right)-g_{i}\left(t_{1}, x\left(t_{2}\right)\right)\right|+\left|g_{i}\left(t_{1}, x\left(t_{2}\right)\right)-g_{i}\left(t_{2}, x\left(t_{2}\right)\right)\right| \\
& \leq C_{i}\left|x\left(t_{1}\right)-x\left(t_{2}\right)\right|^{d_{i}}+\omega\left(g_{i}, \varepsilon\right) \\
& \leq C_{i} \omega(x, \varepsilon)^{d_{i}}+\omega\left(g_{i}, \varepsilon\right),
\end{aligned}
$$

where

$$
\omega\left(g_{i}, \varepsilon\right)=\sup \left\{\left|g_{i}(t, x)-g_{i}(s, x)\right|: t, s \in I,|t-s| \leq \varepsilon, x \in[-r, r]\right\} .
$$

Therefore,

$$
(I) \leq \frac{\left(C_{i} \omega(x, \varepsilon)^{d_{i}}+\omega\left(g_{i}, \varepsilon\right)\right) \varphi_{i}\left(r^{e_{i}}\right)}{\Gamma_{q}\left(\alpha_{i}+1\right)} .
$$

- Estimate of (II). First, observe that

$$
\left|g_{i}\left(t_{2}, x\left(t_{2}\right)\right)\right| \leq\left|g_{i}\left(t_{2}, x\left(t_{2}\right)\right)-g_{i}\left(t_{2}, 0\right)\right|+g_{i}^{*} .
$$

Therefore, by Assumption (A2),

$$
\left|g_{i}\left(t_{2}, x\left(t_{2}\right)\right)\right| \leq C_{i} r^{d_{i}}+g_{i}^{*}
$$

Next, we have

$$
\begin{aligned}
& \left|\int_{0}^{t_{1}}\left(t_{1}-q s\right)^{\left(\alpha_{i}-1\right)} u_{i}(s, x(s)) d_{q} s-\int_{0}^{t_{2}}\left(t_{2}-q s\right)^{\left(\alpha_{i}-1\right)} u_{i}(s, x(s)) d_{q} s\right| \\
& \quad=(1-q) \sum_{n=0}^{\infty} q^{n}\left(1-q^{n+1}\right)^{\left(\alpha_{i}-1\right)}\left|t_{1}^{\alpha_{i}} u_{i}\left(q^{n} t_{1}, x\left(q^{n} t_{1}\right)\right)-t_{2}^{\alpha_{i}} u_{i}\left(q^{n} t_{2}, x\left(q^{n} t_{2}\right)\right)\right| .
\end{aligned}
$$

On the other hand,

$$
\begin{aligned}
& \left|t_{1}^{\alpha_{i}} u_{i}\left(q^{n} t_{1}, x\left(q^{n} t_{1}\right)\right)-t_{2}^{\alpha_{i}} u_{i}\left(q^{n} t_{2}, x\left(q^{n} t_{2}\right)\right)\right| \\
& \leq t_{1}^{\alpha_{i}}\left|u_{i}\left(q^{n} t_{1}, x\left(q^{n} t_{1}\right)\right)-u_{i}\left(q^{n} t_{1}, x\left(q^{n} t_{2}\right)\right)\right| \\
& \quad+\left|t_{1}^{\alpha_{i}} u_{i}\left(q^{n} t_{1}, x\left(q^{n} t_{2}\right)\right)-t_{2}^{\alpha_{i}} u_{i}\left(q^{n} t_{2}, x\left(q^{n} t_{2}\right)\right)\right|
\end{aligned}
$$




$$
\begin{aligned}
& \leq \varphi_{i}\left(\left|x\left(q^{n} t_{1}\right)-x\left(q^{n} t_{2}\right)\right|^{e_{i}}\right)+A_{i}(\varepsilon) \\
& \leq \varphi_{i}\left(\omega(x, \varepsilon)^{e_{i}}\right)+A_{i}(\varepsilon)
\end{aligned}
$$

where

$$
A_{i}(\varepsilon)=\sup \left\{\left|H(\tau, s, x)-H\left(\tau^{\prime}, s^{\prime}, x\right)\right|: \tau, s, \tau^{\prime}, s^{\prime} \in I,\left|\tau-\tau^{\prime}\right| \leq \varepsilon,\left|s-s^{\prime}\right| \leq \varepsilon, x \in[-r, r]\right\}
$$

and

$$
H(\tau, s, x)=\tau^{\alpha_{i}} u_{i}(s, x), \quad(\tau, s, x) \in I \times I \times \mathbb{R} .
$$

As a consequence, we get

$$
\begin{aligned}
& \left|\int_{0}^{t_{1}}\left(t_{1}-q s\right)^{\left(\alpha_{i}-1\right)} u_{i}(s, x(s)) d_{q} s-\int_{0}^{t_{2}}\left(t_{2}-q s\right)^{\left(\alpha_{i}-1\right)} u_{i}(s, x(s)) d_{q} s\right| \\
& \quad \leq \varphi_{i}\left(\omega(x, \varepsilon)^{e_{i}}\right)+A_{i}(\varepsilon) .
\end{aligned}
$$

Then

$$
(I I) \leq \frac{\left(C_{i} r^{d_{i}}+g_{i}^{*}\right)\left(\varphi_{i}\left(\omega(x, \varepsilon)^{e_{i}}\right)+A_{i}(\varepsilon)\right)}{\Gamma_{q}\left(\alpha_{i}\right)}
$$

Now, combining (4.4) with (4.5), we get

$$
\omega\left(T_{i} x, \varepsilon\right) \leq \omega\left(f_{i}, \varepsilon\right)+\frac{\left(C_{i} \omega(x, \varepsilon)^{d_{i}}+\omega\left(g_{i}, \varepsilon\right)\right) \varphi_{i}\left(r^{e_{i}}\right)}{\Gamma_{q}\left(\alpha_{i}+1\right)}+\frac{\left(C_{i} r^{d_{i}}+g_{i}^{*}\right)\left(\varphi_{i}\left(\omega(x, \varepsilon)^{e_{i}}\right)+A_{i}(\varepsilon)\right)}{\Gamma_{q}\left(\alpha_{i}\right)} .
$$

Therefore,

$$
\begin{aligned}
\omega\left(T_{i} M, \varepsilon\right) \leq & \omega\left(f_{i}, \varepsilon\right)+\frac{\left(C_{i} \omega(M, \varepsilon)^{d_{i}}+\omega\left(g_{i}, \varepsilon\right)\right) \varphi_{i}\left(r^{e_{i}}\right)}{\Gamma_{q}\left(\alpha_{i}+1\right)} \\
& +\frac{\left(C_{i} r^{d_{i}}+g_{i}^{*}\right)\left(\varphi_{i}\left(\omega(M, \varepsilon)^{e_{i}}\right)+A_{i}(\varepsilon)\right)}{\Gamma_{q}\left(\alpha_{i}\right)} .
\end{aligned}
$$

Passing to the limit as $\varepsilon \rightarrow 0$, we obtain

$$
\mu\left(T_{i} M\right) \leq \frac{C_{i} \mu(M)^{d_{i}} \varphi_{i}\left(r^{e_{i}}\right)}{\Gamma_{q}\left(\alpha_{i}+1\right)}+\frac{\left(C_{i} r^{d_{i}}+g_{i}^{*}\right) \varphi_{i}\left(\mu(M)^{e_{i}}\right)}{\Gamma_{q}\left(\alpha_{i}\right)} .
$$

Then

$$
\mu\left(T_{i} M\right) \leq \max \left\{\frac{C_{i} \varphi_{i}\left(r^{e_{i}}\right)}{\Gamma_{q}\left(\alpha_{i}+1\right)}, \frac{\left(C_{i} r^{d_{i}}+g_{i}^{*}\right)}{\Gamma_{q}\left(\alpha_{i}\right)}\right\}\left(\mu(M)^{d_{i}}+\varphi_{i}\left(\mu(M)^{e_{i}}\right)\right) .
$$

By Assumption (A6) we obtain

$$
\mu\left(T_{i} M\right) \leq \lambda_{i} \max \left\{\frac{C_{i} \varphi_{i}\left(r^{e_{i}}\right)}{\Gamma_{q}\left(\alpha_{i}+1\right)}, \frac{\left(C_{i} r^{d_{i}}+g_{i}^{*}\right)}{\Gamma_{q}\left(\alpha_{i}\right)}\right\} \mu(M),
$$

which proves the desired result. 
Now, we are able to state and prove our main result.

Theorem 4.5 Suppose that Assumptions (A1)-(A6) are satisfied. If

$$
r^{\frac{N-1}{N}} \sum_{i=1}^{N} \lambda_{i} \max \left\{\frac{C_{i} \varphi_{i}\left(r^{e_{i}}\right)}{\Gamma_{q}\left(\alpha_{i}+1\right)}, \frac{\left(C_{i} r^{d_{i}}+g_{i}^{*}\right)}{\Gamma_{q}\left(\alpha_{i}\right)}\right\}<1
$$

then equation (1.1) has at least one solution $x^{*} \in \overline{B\left(0_{E}, r\right)}$.

Proof Observe that $x \in \overline{B\left(0_{E}, r\right)}$ is a solution to equation (1.1) if and only if $x$ is a solution to (3.3), where $D=\overline{B\left(0_{E}, r\right)}$, and $T$ is given by (4.2). In order to prove our existence result, we have to check that all the assumptions of Theorem 3.5 are satisfied. By Lemma 4.3, for all $i=1, \ldots, N, T_{i}: \overline{B\left(0_{E}, r\right)} \rightarrow E$ is a continuous operator. By Lemma 4.2, for all $i=1, \ldots, N$, $T_{i} \overline{B\left(0_{E}, r\right)}$ is bounded. Moreover, for all $x \in \overline{B\left(0_{E}, r\right)}$, we have

$$
\|T x\|_{E}=\left\|\prod_{i=1}^{N} T_{i} x\right\|_{E} \leq \prod_{i=1}^{N}\left\|T_{i} x\right\|_{E} \leq \prod_{i=1}^{N} r^{1 / N}=r .
$$

Therefore, $T \overline{B\left(0_{E}, r\right)} \subset \overline{B\left(0_{E}, r\right)}$. By Lemma 4.4 condition (iv) of Theorem 3.5 is satisfied with

$$
k_{i}=\lambda_{i} \max \left\{\frac{C_{i} \varphi_{i}\left(r^{e_{i}}\right)}{\Gamma_{q}\left(\alpha_{i}+1\right)}, \frac{\left(C_{i} r^{d_{i}}+g_{i}^{*}\right)}{\Gamma_{q}\left(\alpha_{i}\right)}\right\}, \quad i=1, \ldots, N
$$

On the other hand, from Lemma 4.2 and (4.6) we have

$$
\sum_{i=1}^{q} k_{i} \prod_{j=1, j \neq i}^{q}\left\|T_{j} \overline{B\left(0_{E}, r\right)}\right\| \leq r^{\frac{N-1}{N}} \sum_{i=1}^{N} \lambda_{i} \max \left\{\frac{C_{i} \varphi_{i}\left(r^{e_{i}}\right)}{\Gamma_{q}\left(\alpha_{i}+1\right)}, \frac{\left(C_{i} r^{d_{i}}+g_{i}^{*}\right)}{\Gamma_{q}\left(\alpha_{i}\right)}\right\}<1
$$

Therefore, all conditions of Theorem 3.5 are satisfied, and the desired result follows.

We end the paper with the following illustrative example.

Example Consider the functional equation

$$
x(t)=\frac{t}{32}+\left[\frac{3}{2}\right]_{\frac{1}{2}}\left(\frac{t}{2}+\frac{x(t)}{4}\right) \int_{0}^{t}(t-s / 2)^{(1 / 2)} \frac{x(s)}{\left(2+s^{2}\right)} d_{q} s, \quad t \in[0,1] .
$$

Equation (4.7) is a a particular case of equation (1.1) with

$$
\begin{aligned}
& N=1, \quad q=\frac{1}{2}, \quad \alpha_{1}=\frac{3}{2}, \quad f_{1}(t)=\frac{t}{32}, \\
& g_{1}(t, x)=\left(\frac{t}{2}+\frac{x}{4}\right) \Gamma_{\frac{1}{2}}\left(\frac{5}{2}\right), \quad u_{1}(t, x)=\frac{x}{2+t^{2}} .
\end{aligned}
$$

Obviously, the functions $f_{1}:[0,1] \rightarrow \mathbb{R}$ and $g_{1}, u_{1}:[0,1] \times \mathbb{R} \rightarrow \mathbb{R}$ are continuous. Then Assumption (A1) of Theorem 4.5 is satisfied. For all $(t, x, y) \in[0,1] \times \mathbb{R} \times \mathbb{R}$, we have

$$
\left|g_{1}(t, x)-g_{1}(t, y)\right| \leq \frac{\Gamma_{\frac{1}{2}}\left(\frac{5}{2}\right)}{4}|x-y|
$$


Therefore, Assumption (A2) is satisfied with

$$
C_{1}=\frac{\Gamma_{\frac{1}{2}}\left(\frac{5}{2}\right)}{4}, \quad d_{1}=1
$$

For all $(t, x, y) \in[0,1] \times \mathbb{R} \times \mathbb{R}$, we have

$$
\left|u_{1}(t, x)-u_{1}(t, y)\right| \leq \frac{1}{2}|x-y| .
$$

Moreover, $u_{1}(t, 0)=0$ for all $t \in[0,1]$. Therefore, Assumptions (A3) and (A4) are satisfied with

$$
\varphi_{1}(t)=\frac{t}{2}, \quad e_{1}=1
$$

Note that, in this case, we have

$$
\left\|f_{1}\right\|_{E}=\frac{1}{32}, \quad g_{1}^{*}=\frac{\Gamma_{\frac{1}{2}}\left(\frac{5}{2}\right)}{2} .
$$

It is not difficult to check that Assumption (A5) is satisfied for every $r \geq \frac{1}{20}$. For every $t \geq 0$, we have

$$
t^{d_{1}}+\varphi\left(t^{e_{1}}\right)=\frac{3}{2} t
$$

Then Assumption (A6) is satisfied with $\lambda_{1}=\frac{3}{2}$. Moreover, for $r=\frac{1}{20}$, we have

$$
\lambda_{1} \max \left\{\frac{C_{1} \varphi_{1}\left(r^{e_{1}}\right)}{\Gamma_{q}\left(\alpha_{1}+1\right)}, \frac{\left(C_{1} r^{d_{1}}+g_{1}^{*}\right)}{\Gamma_{q}\left(\alpha_{1}\right)}\right\}=0.99391166196<1 .
$$

By Theorem 4.5 we deduce that equation (4.7) admits at least one solution $x^{*} \in \overline{B\left(0_{E}, \frac{1}{20}\right)}$.

\section{Competing interests}

The authors declare to have no competing interests.

\section{Authors' contributions}

Both authors contributed equally in writing this paper. Both authors read and approved the final manuscript.

\section{Acknowledgements}

The second author extends his appreciation to Distinguished Scientist Fellowship Program (DSFP) at King Saud University (Saudi Arabia).

Received: 8 September 2016 Accepted: 4 January 2017 Published online: 18 January 2017

\section{References}

1. Brestovanska, E: Qualitative behaviour of an integral equation related to some epidemic model. Demonstr. Math. 36(3), 603-609 (2003)

2. Gripenberg, G: On some epidemic models. Q. Appl. Math. 39, 317-327 (1981)

3. Olaru, IM: Generalization of an integral equation related to some epidemic models. Carpath. J. Math. 26, $92-96$ (2010)

4. Agarwal, RP: Certain fractional $q$-integrals and q-derivatives. Proc. Camb. Philos. Soc. 66, 365-370 (1969)

5. Aghajani, A, Allahyari, R, Mursaleen, M: A generalization of Darbo's theorem with application to the solvability of systems of integral equations. J. Comput. Appl. Math. 260, 68-77 (2014)

6. Aghajani, A, Banas, J, Jalilian, Y: Existence of solutions for a class of nonlinear Volterra singular integral equations. Comput. Math. Appl. 62, 1215-1227 (2011)

7. Aghajani, A, Mursaleen, M, Shole Haghighi, A: Fixed point theorems for Meir-Keeler condensing operators via measure of noncompactness. Acta Math. Sci. 35(3), 552-566 (2015) 
8. Aghajani, A, Pourhadi, E, Trujillo, Jj: Application of measure of noncompactness to a Cauchy problem for fractional differential equations in Banach spaces. Fract. Calc. Appl. Anal. 16(4), 962-977 (2013)

9. Banas, J, Caballero, J, Rocha, J, Sadarangani, K: Monotonic solutions of a class of quadratic integral equations of Volterra type. Comput. Math. Appl. 49, 943-952 (2005)

10. Banas, J, Goebel, K: Measures of Noncompactness in Banach Spaces. Lect. Notes Pure Appl. Math., vol. 60. Dekker, New York (1980)

11. Banas, J, Martinon, A: Monotonic solutions of a quadratic integral equation of Volterra type. Comput. Math. Appl. 47, 271-279 (2004)

12. Banas, J, Mursaleen, M: Sequence Spaces and Measures of Noncompactness with Applications to Differential and Integral Equations. Springer, New Dehli (2014)

13. Banas, J, Olszowy, L: On a class of measures of non-compactness in Banach algebras and their application to nonlinear integral equations. Z. Anal. Anwend. 28, 475-498 (2009)

14. Banaś, J, O'Regan, D: On existence and local attractivity of solutions of a quadratic integral equation of fractional order. J. Math. Anal. Appl. 345, 573-582 (2008)

15. Darwish, MA, Sadarangani, K: On a quadratic integral equation with supremum involving Erdélyi-Kober fractional order. Math. Nachr. 288(5-6), 566-576 (2015)

16. Jleli, M, Mursaleen, M, Samet, B: Q-Integral equations of fractional orders. Electron. J. Differ. Equ. 2016, 17 (2016)

17. Jackson, FH: On q-functions and a certain difference operator. Trans. R. Soc. Edinb. 46, 253-281 (1908)

18. Jackson, FH: On q-definite integrals. Q. J. Pure Appl. Math. 41, 193-203 (1910)

19. Abdeljawad, T, Baleanu, D: Caputo $q$-fractional initial value problems and a $q$-analogue Mittag-Leffler function. Commun. Nonlinear Sci. Numer. Simul. 16, 4682-4688 (2011)

20. Ahmad, B, Nieto, J: Basic theory of nonlinear third-order q-difference equations and inclusions. Math. Model. Anal. 18(1), 122-135 (2013)

21. Annaby, MH, Mansour, ZS: q-Fractional Calculus and Equations. Lecture Notes in Mathematics, vol. 2056. Springer, Berlin (2012)

22. Atici, FM, Eloe, PW: Fractional q-calculus on a time scale. J. Nonlinear Math. Phys. 14(3), 333-344 (2007)

23. Ernst, T: The History of $q$-Calculus and a New Method. U. U. D. M. Report 2000:16, Department of Mathematics, Uppsala University (2000)

24. Jarad, F, Abdeljawad, T, Baleanu, D: Stability of $q$-fractional non-autonomous systems. Nonlinear Anal., Real World Appl. 14(1), 780-784 (2013)

25. Kac, V, Cheung, P: Quantum Calculus. Springer Science \& Business Media (2002)

26. Wu, GC, Baleanu, D: New applications of the variational iteration method-from differential equations to $q$-fractional difference equations. Adv. Differ. Equ. 2013, 21 (2013)

27. Ferreira, R: Nontrivial solutions for fractional $q$-difference boundary value problems. Electron. J. Qual. Theory Differ Equ. 2010, $70(2010)$

28. Darbo, G: Punti uniti in transformazioni a condomino non compatto. Rend. Semin. Mat. Univ. Padova 24, 84-92 (1955)

\section{Submit your manuscript to a SpringerOpen ${ }^{\circ}$ journal and benefit from:}

- Convenient online submission

Rigorous peer review

- Immediate publication on acceptance

- Open access: articles freely available online

- High visibility within the field

- Retaining the copyright to your article 